\title{
A transport-theoretic analysis of pulse propagation through ocean sediments
}

\author{
loannis M. Besieris and Werner E. Kohler \\ Virginia Polytechnic Institute and State University, Blacksburg, Virginia 24061 \\ Herbert Freese \\ Areté Associates, P. O. Box 2951, Eads Street Station, Arlington, Virginia 22202
}

(Received 1 August 1981; accepted for publication 3 June 1982)

The reflection of pulsed acoustic plane waves from ocean sedimentary layers is studied using a stochastic transport theory originally introduced by Barabanenkov et al. [Izv. Vyssh. Uchebn. Zaved., Radiofiz 15, 1852 (1972)]. The sediments are assumed to be a random medium in which the density and sound speed undergo small, highly laminated (pancakelike) fluctuations.

Although the problem is formulated in a general context, the predictions of the theory are fully evaluated only in the special case of normal incidence and no refracting profile. However, even with these approximations, some reasonable qualitative agreement of theoretical predictions with measured data is achieved.

PACS numbers: 43.30.Dr, 92.10.Vz, 43.20.Bi, 43.20.Fn

\section{INTRODUCTION}

The abyssal plains are extensive regions of the ocean bottom consisting essentially of a thick sediment layer overlying a rock basement. ${ }^{1}$ Sediment density and sound speed increase slowly (in a nominally linear manner) as depth into the layer increases. Superposed upon this gross variation, however, is a small, highly anisotropic fluctuation structure. The density and sound speed fluctuate very rapidly in the depth direction while in the transverse plane it is conjectured that the rate of variation is much slower.

We study the reflection of pulsed acoustic plane waves from such a refracting, highly anisotropic (pancakelike) random medium. For simplicity, the sediment layer is assumed to be infinitely thick; if desired, a rock basement can be introduced into the model without difficulty. The analysis uses a stochastic transport theory that was originally introduced by Barabanenkov et al. ${ }^{2}$ and subsequently extended to the two-frequency context needed for the study of pulse propagation. $^{3}$

In Sec. I we formulate the basic problem, derive a stochastic Helmholtz equation and formulate a model for the random density and sound-speed fluctuations. Once the Helmholtz equation (with an associated refractive index) is obtained, dissipation is introduced into the model phenomenologically. In Sec. II the structure and physical content of the two-frequency stochastic transport theory are discussed while in Sec. III the simplifications and approximations permitted by the severe anisotropy of the random field are developed. In the case where refraction is neglected, these approximations lead to Schuster equations ${ }^{4}$ for the incoherent intensities. In Sec. IV we formulate an expression for the quantity of interest, which is basically the mutual coherence function for the complex pressure. The problem is then solved for the special case of normal incidence and no refraction. In the course of obtaining this solution, we exploit some ideas of Keller ${ }^{5}$ to derive appropriate boundary conditions at the water-sediment interface. The predictions of the theory for the special case considered are numerically evaluated and qualitatively compared with experimental data in Sec. V. Section VI summarizes our conclusions.

Restricting our attention to normal incidence is largely a matter of convenience. Appendix A shows how the results obtained can be extended to accommodate oblique incidence. Neglect of refraction, however, is a significant simplification. Some features of the refracting model, which is currently being studied, are discussed in Sec. III. Lastly, Appendix $B$ points out that the sequence of approximations that we have made in developing the model has preserved the property of power conservation.

\section{PROBLEM FORMULATION}

The problem to be considered is summarized in Fig. 1. A pulsed plane wave is incident upon a sediment half-space from water, whose density and sound speed are constant. In the sediment, the density and sound speed are assumed to have mean values that depend only upon the depth variable $z$; for simplicity, a common linear gradient is adopted. The random zero mean fluctuations, however, depend upon all the spatial coordinates (in a highly anisotropic manner to be specified). Therefore the density and sound speed are defined

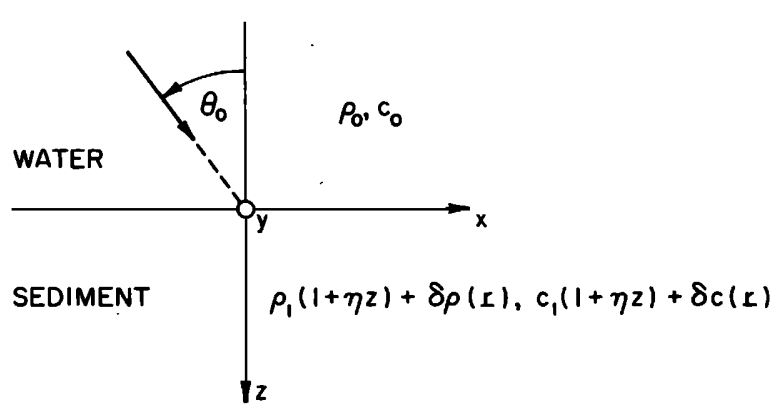

FIG. 1. Problem configuration. 
as

$$
\begin{array}{r}
\rho(\mathbf{r})= \begin{cases}\rho_{0}, & z<0, \\
\rho_{1}(1+\eta z)+\delta \rho(\mathbf{r}), & z \geqslant 0,\end{cases} \\
c(\mathbf{r})= \begin{cases}c_{0}, & z<0, \\
c_{1}(1+\eta z)+\delta c(\mathbf{r}), & z \geqslant 0,\end{cases}
\end{array}
$$

with $\langle\delta \rho\rangle=0$ and $\langle\delta c\rangle=0$, where $\langle\cdot\rangle$ denotes expected value. (We suppress dependence upon a random index.) Note that the mean density and sound speed will generally be discontinuous at the interface (if $\rho_{1} \neq \rho_{0}$ and $c_{1} \neq c_{0}$ ).

With $p(\mathbf{r}, t)$ and $v(\mathbf{r}, t)$ denoting pressure and velocity, the basic equations are ${ }^{6}$

$$
\begin{aligned}
& \frac{\partial}{\partial t} p(\mathbf{r}, t)+\rho(\mathbf{r}) c^{2}(\mathbf{r}) \nabla \cdot \mathbf{v}(\mathbf{r}, t)=0 \\
& \frac{\partial}{\partial t} \nabla(\mathbf{r}, t)+\rho^{-1}(\mathbf{r}) \nabla p(\mathbf{r}, t)=0
\end{aligned}
$$

with pressure and normal velocity component continuous across the interface. We introduce next the Fourier transforms

$$
\begin{aligned}
& \hat{p}(\mathbf{r}, \omega) \equiv \frac{1}{2 \pi} \int_{-\infty}^{\infty} p(\mathbf{r}, t) e^{i \omega t} d t, \\
& \hat{\mathbf{v}}(\mathbf{r}, \omega) \equiv \frac{1}{2 \pi} \int_{-\infty}^{\infty} \mathrm{v}(\mathbf{r}, t) e^{i \omega t} d t .
\end{aligned}
$$

In terms of these transforms, Eqs. (2) become

$$
-i \omega \hat{p}+\rho c^{2} \nabla \cdot \hat{v}=0, \quad-i \omega \hat{\mathbf{v}}+\rho^{-1} \nabla \hat{p}=0 .
$$

Using Eqs. (4), we obtain the following equation for the complex transformed pressure $\hat{p}$ :

$$
\nabla \cdot\left(\rho^{-1} \nabla \hat{p}\right)+\left(\omega^{2} / \rho c^{2}\right) \hat{p}=0 .
$$

Let $k \equiv \omega / c_{0}$, i.e., we reference wavenumber to $c_{0}$. We also define the following dimensionless density and sound speed:

$$
\begin{gathered}
\tilde{\rho}(\mathbf{r}) \equiv \rho(\mathbf{r}) / \rho_{0}= \begin{cases}1, & z<0, \\
\tilde{\rho}_{1}(1+\eta z)+\delta \tilde{\rho}(\mathbf{r}), & z \geqslant 0,\end{cases} \\
\tilde{c}(\mathbf{r}) \equiv c(\mathbf{r}) / c_{0}= \begin{cases}1, & z<0, \\
\tilde{c}_{1}(1+\eta z)+\delta \tilde{c}(\mathbf{r}), & z \geqslant 0 .\end{cases}
\end{gathered}
$$

Following Brekovskikh, ${ }^{6}$ we transform (5) into a Helmholtz equation by making the change of dependent variable

$$
u(\mathbf{r}, k) \equiv \rho^{-1 / 2}(\mathbf{r}) \hat{p}(\mathbf{r}, \omega) .
$$

The resulting equation for $u$ is given as follows:

$$
\Delta u+\left(\frac{k^{2}}{\tilde{c}^{2}}+\frac{1}{2 \tilde{\rho}} \Delta \tilde{\rho}-\frac{3}{4 \tilde{\rho}^{2}}(\nabla \tilde{\rho})^{2}\right) u=0 .
$$

We assume that a single side-band narrow bandwidth pulse, with carrier wavenumber $k_{0} \equiv \omega_{0} / c_{0}$, is transmitted. Also, we recall that the random fluctuations $\delta \tilde{\rho}, \delta \tilde{c}$ are small in amplitude and highly anisotropic (i.e., slowly varying in the transverse direction but very rapidly varying in the $z$ direction). Guided by these observations, we recast (8) in the form

$$
\Delta u+k^{2} \epsilon(\mathrm{r}, k) u=0,
$$

where $\epsilon(r, k) \equiv 1$ for $z<0$, while for $z \geqslant 0$

$$
\begin{aligned}
\epsilon(\mathbf{r}, k) \cong & \epsilon\left(\mathbf{r}, k_{0}\right) \cong \tilde{c}_{1}^{-2}(1+\eta z)^{-2}-2 \tilde{c}_{1}^{-3}(1+\eta z)^{-3} \\
& \times \delta \tilde{c}(\mathbf{r})+\left[2 k_{0}^{2} \tilde{\rho}_{1}(1+\eta z)\right]^{-1} \frac{\partial^{2}}{\partial z^{2}}[\delta \tilde{\rho}(\mathbf{r})] \\
& -\frac{3}{4}\left[k_{0} \tilde{\rho}_{1}(1+\eta z)\right]^{-2}\left[\left(\eta \tilde{\rho}_{1}\right)^{2}\right. \\
& \left.+2 \eta \tilde{\rho}_{1} \frac{\partial}{\partial z}[\delta \tilde{\rho}(\mathbf{r})]+\left(\left(\frac{\partial}{\partial z}[\delta \tilde{\rho}(\mathbf{r})]\right)^{2}\right)\right] .
\end{aligned}
$$

In obtaining (10), the following approximations were made:

(i) Wavenumber dependence was reduced to the carrier wavenumber;

(ii) $\tilde{c}^{-2}(\mathbf{r}) \cong \tilde{c}_{1}^{-2}(1+\eta z)^{-2}-2 \tilde{c}_{1}^{-3}(1+\eta z)^{-3} \delta \tilde{c}(\mathbf{r})$;

(iii) $\nabla \tilde{\rho}(\mathbf{r}) \cong\left(\eta \tilde{\rho}_{1}+\frac{\partial}{\partial z}[\delta \tilde{\rho}(\mathbf{r})]\right) \mathbf{z}_{0}$ (with $\mathbf{z}_{0}$ a $z$-directed unit vector);

(iv) $\Delta \tilde{\rho}(\mathbf{r}) \cong \frac{\partial^{2}}{\partial z^{2}}[\delta \tilde{\rho}(\mathbf{r})]$;

(v) $\tilde{\rho}^{-1}(\mathbf{r})$ and $\tilde{\rho}^{-2}(r)$ have been approximated by $\langle\tilde{\rho}(\mathbf{r})\rangle^{-1}$ and $\langle\tilde{\rho}(\mathbf{r})\rangle^{-2}$;

(vi) The fluctuations in $\left(\frac{\partial}{\partial z}[\delta \tilde{\rho}(\mathbf{r})]\right)^{2}$ have been neglected.

If (9) is viewed in an electromagnetic context, $\epsilon$ is called a relative dielectric constant. For convenience, we shall use the same terminology in the present acoustic context. When decomposed into mean value and fluctuations, (10) assumes the form

$$
\begin{aligned}
\epsilon\left(\mathbf{r}, k_{0}\right)= & \epsilon_{01}\left(k_{0}\right)(1+\eta z)^{-2}+\delta \epsilon\left(\mathbf{r}, k_{0}\right), \quad z \geqslant 0 ; \\
\epsilon_{01}\left(k_{0}\right) \equiv & \tilde{c}_{1}^{-2}-\frac{3}{4}\left(k_{0} \tilde{\rho}_{1}\right)^{-1}\left[\left(\eta \tilde{\rho}_{1}\right)^{2}+\left(\left(\frac{\partial}{\partial z}[\delta \tilde{\rho}(\mathbf{r})]\right)^{2}\right)\right], \\
\delta \epsilon\left(\mathbf{r}, k_{0}\right) \equiv & -2 \tilde{c}_{1}^{-3}(1+\eta z)^{-3} \delta \tilde{c}(\mathbf{r})+\left[2 k_{0}^{2} \tilde{\rho}_{1}(1+\eta z)\right] \\
& \times \frac{\partial^{2}}{\partial z^{2}}[\delta \tilde{\rho}(\mathbf{r})]-\frac{3}{4}\left[k_{0} \tilde{\rho}_{1}(1+\eta z)\right]^{-2} \\
& \times 2 \eta \tilde{\rho}_{1} \frac{\partial}{\partial z}[\delta \tilde{\rho}(\mathbf{r})],
\end{aligned}
$$

where we have assumed $\left\langle\left[(\partial / \partial z)\{[\delta \tilde{\rho}(\mathbf{r})]\}^{2}\right\rangle \cong\right.$ constant.

In order to implement the stochastic transport theory, it will be necessary to determine the two-point correlation function for the zero-mean fluctuation $\delta \epsilon\left(r, k_{0}\right)$. We begin by assuming the following correlation structure for density and sound-speed fluctuations:

$$
\begin{aligned}
\left\langle\delta \tilde{\rho}\left(\mathbf{r}_{1}\right) \delta \tilde{\rho}\left(\mathbf{r}_{2}\right)\right\rangle= & \sigma_{\rho \rho} \exp \left[-\beta\left[\left(x_{1}-x_{2}\right)^{2}+\left(y_{1}-y_{2}\right)^{2}\right]^{1 / 2}\right\} \\
& \times \exp \left[-\left(z_{1}-z_{2}\right)^{2} / 2 l_{z}^{2}\right], \\
\left\langle\delta \tilde{\rho}\left(\mathbf{r}_{1}\right) \delta \tilde{c}\left(\mathbf{r}_{2}\right)\right\rangle= & \sigma_{\rho c} \exp \left[-\beta\left[\left(x_{1}-x_{2}\right)^{2}+\left(y_{1}-y_{2}\right)^{2}\right]^{1 / 2}\right\} \\
& \times \exp \left[-\left(z_{1}-z_{2}\right)^{2} / 2 l_{z}^{2}\right], \\
\left\langle\delta \tilde{c}\left(\mathbf{r}_{1}\right) \delta \tilde{c}\left(\mathbf{r}_{2}\right)\right\rangle= & \sigma_{c c} \exp \left\{-\beta\left[\left(x_{1}-x_{2}\right)^{2}+\left(y_{1}-y_{2}\right)^{2}\right]^{1 / 2}\right\} \\
& \times \exp \left[-\left(z_{1}-z_{2}\right)^{2} / 2 l_{z}^{2}\right] .
\end{aligned}
$$

In (12) $\beta \equiv l_{T}^{-1}$ is the reciprocal of the transverse correlation length while $l_{z}$ is the correlation length in the depth direction. For the highly anisotropic (pancakelike) medium being considered, both $\beta$ and $l_{z}$ will be assumed to be small parameters. (Carrier wavelength serves as our basic length scale.) The second moments $\sigma_{\rho \rho}, \sigma_{\rho c}$, and $\sigma_{c c}$ will be taken to be constants. Moreover, we shall assume that the same underly- 
ing physical mechanism (and hence the same basic underlying random field) determines both the density and soundspeed fluctuations. This assumption leads to the relation $\sigma_{\rho c}$ $=\left(\sigma_{\rho \rho} \sigma_{c c^{3}}\right)^{1 / 2}$.

We define the following sum and difference coordinates:

$$
\begin{aligned}
& \mathbf{R}=X \mathbf{x}_{0}+Y \mathbf{y}_{0}+Z \mathbf{z}_{0} \equiv\left(\mathbf{r}_{1}+\mathbf{r}_{2}\right) / 2, \\
& \mathbf{r}=x \mathbf{x}_{0}+y \mathbf{y}_{0}+z \mathbf{z}_{0} \equiv \mathbf{r}_{1}-\mathbf{r}_{2} .
\end{aligned}
$$

In terms of these coordinates, the correlation function for the dielectric constant fluctuations becomes

$$
\begin{aligned}
\left\langle\delta \epsilon\left(\mathbf{r}_{1}, k_{0}\right) \delta\right. & \left.\epsilon\left(\mathbf{r}_{2}, k_{0}\right)\right\rangle \cong B_{0}(\eta Z) \exp \left[-\beta\left(x^{2}+y^{2}\right)^{1 / 2}\right] \\
x \delta(z), \quad Z & \geqslant 0, \\
B_{0}(\eta Z) \equiv & \sqrt{2 \pi} l_{z}\left\{4 \tilde{c}_{1}^{-6}(1+\eta Z)^{-6} \sigma_{c c}\right. \\
& +2\left(\tilde{c}_{1}^{3} k_{0}^{2} l_{z}^{2} \tilde{\rho}_{1}\right)^{-1}(1+\eta Z)^{-4} \sigma_{\rho c} \\
& +\left(2 k_{0}^{2} \tilde{\rho}_{1}\right)^{-2}\left[3 l_{z}^{-4}(1+\eta Z)^{-2}\right. \\
& \left.\left.+9 l_{z}^{-2} \eta^{2}(1+\eta Z)^{-4}\right] \sigma_{\rho \rho}\right\} .
\end{aligned}
$$

Equations (14) result from the substitution of (12) into (11) and the subsequent idealization of the depth dependence to $\delta$ correlation.

As a final step in our modeling of the relative dielectric constant, we shall introduce dissipation phenomenologically. Since absorption in the sediment is small, ${ }^{7}$ we shall add a small nonrandom imaginary component to the mean dielectric constant. Our final model for the dielectric constant is

$$
\begin{aligned}
& \epsilon\left(\mathbf{r}, k_{0}\right)=\left\langle\epsilon\left(\mathbf{r}, k_{0}\right)\right\rangle+\delta \epsilon\left(\mathbf{r}, k_{0}\right) ; \\
& \left\langle\epsilon\left(\mathbf{r}, k_{0}\right)\right\rangle= \begin{cases}1, & z<0, \\
\epsilon_{01}\left(k_{0}\right)(1+\eta z)^{-2}+i \epsilon_{02}\left(\eta z, k_{0}\right), & z \geqslant 0,\end{cases} \\
& \epsilon_{01}\left(k_{0}\right) \equiv \tilde{c}_{1}^{-2}-\frac{3}{4}\left(k_{0} \tilde{\rho}_{1}\right)^{-2}\left[\left(\eta \tilde{\rho}_{1}\right)^{2}+l_{z}^{-2} \sigma_{\rho \rho}\right], \\
& \delta \epsilon\left(\mathbf{r}, k_{0}\right)=0, \quad z<0, \\
& \left\langle\delta \epsilon\left(\mathbf{r}, k_{0}\right)\right\rangle=0, \quad z \geqslant 0, \\
& \left\langle\delta \epsilon\left(\mathbf{R}+\frac{1}{2} \mathbf{r}, k_{0}\right) \delta \epsilon\left(\mathbf{R}-\frac{1}{2} \mathbf{r}, k_{0}\right)\right\rangle=B_{0}(\eta Z) e^{-\beta \rho} \delta(z), \quad Z \geqslant 0,
\end{aligned}
$$

where $\rho \equiv\left(x^{2}+y^{2}\right)^{1 / 2}$ and $B_{0}$ is defined in (14). The dissipation $\epsilon_{02}$ can be any non-negative slowly varying function of depth; in our computations, however, we shall generally assume it constant. [In (15) the symbol $r$ is used to denote both a generic position and also a difference coordinate; the meaning in each case should be clear from the context.]

Remarks: The dielectric constant model that we have evolved [Eqs. (15)] represents a compromise between our needs to both model reality and to compute explicitly. Note that while some dispersive effects have been eliminated by approximating $\epsilon(\mathbf{r}, k) \cong \epsilon\left(r, k_{0}\right)$, the basic dependence will prove very important in relating theory to experimental observations. Note further that the dielectric constant model is valid only over a limited band of frequencies. At sufficiently low frequencies, $\epsilon_{01}\left(k_{0}\right)$ becomes negative causing the model to break down. At sufficiently high frequencies, the assumption that $l_{z}<\lambda$ is no longer true. For the experimental configuration studied, however (i.e., physical constants characteristic of the Hatteras plain and carrier frequencies in the range $700-2000 \mathrm{~Hz}$ ), the model is applicable.

The dominant contribution to $B_{0}$ in (14) arises from the density fluctuations [i.e., the term $(2 \pi)^{1 / 2} l_{z}\left(2 k_{0}^{2} \tilde{\rho}_{1}\right)^{-2} 3 l_{z}^{-4}(1$ $+\eta Z)^{-2} \sigma_{\rho \rho}$. This suggests that density fluctuations play a far more significant role in sediment propagation than comparable sound-speed fluctuations. This conclusion is somewhat paradoxical since the original equations (2) seem to treat density and sound speed on an equal basis. Obviously, much additional modeling work needs to be done in order to test the validity of this conclusion, to eliminate the shortcomings of (15), and to build in more realism (e.g., surface roughness, layering undulations, and compatibility with data such as Tucholke's ${ }^{8}$ which suggests that the fluctuations should have a depth dependence that is basically Poisson in character).

\section{STOCHASTIC TRANSPORT THEORY}

Recall that $u(\mathbf{r}, k)=\rho^{-1 / 2}(\mathbf{r}) \hat{p}(\mathbf{r}, k)$ is the solution of the Helmholtz equation (9), where we now assume that $\epsilon$ is given by (15). Our basic quantity of interest is the mutual coherence function for the complex pressure (for points $r_{1}, r_{2}$ on a receiving array in the water). This expression is given by [cf. Eq. (3)]

$$
\begin{aligned}
\left\langle p\left(\mathbf{r}_{1}, t_{1}\right) p^{*}\left(\mathbf{r}_{2}, t_{2}\right)\right\rangle= & \rho_{0} c_{0}^{2} \int_{-\infty}^{\infty} \int_{-\infty}^{\infty}\left\langle u\left(\mathbf{r}_{1}, k_{1}\right) u^{*}\left(\mathbf{r}_{2}, k_{2}\right)\right\rangle \\
& \times \exp \left[-i c_{0}\left(k_{1} t_{1}-k_{2} t_{2}\right)\right] d k_{1} d k_{2} .
\end{aligned}
$$

Let

$$
\begin{aligned}
& u(\mathbf{r}, k) \equiv \tilde{u}(\mathbf{r}, k)+\delta u(\mathbf{r}, k) \\
& \tilde{u}(\mathbf{r}, k) \equiv\langle u(\mathbf{r}, k)\rangle, \quad\langle\delta u(\mathbf{r}, k)\rangle=0 .
\end{aligned}
$$

Since

$$
\begin{aligned}
\left\langle u\left(\mathbf{r}_{1}, k_{1}\right) u^{*}\left(\mathbf{r}_{2}, k_{2}\right)\right\rangle= & \tilde{u}\left(\mathbf{r}_{1}, k_{1}\right) \tilde{u}^{*}\left(\mathbf{r}_{2}, k_{2}\right) \\
& +\left\langle\delta u\left(\mathbf{r}_{1}, k_{1}\right) \delta u^{*}\left(\mathbf{r}_{2}, k_{2}\right)\right\rangle,
\end{aligned}
$$

the problem is essentially one of solving for the mean or coherent field $\tilde{u}(\mathbf{r}, k)$ at general wavenumber $k$ and the twofrequency incoherent component $\left\langle\delta u\left(\mathbf{r}_{1}, k_{1}\right) \delta u^{*}\left(\mathbf{r}_{2}, k_{2}\right)\right\rangle$. As (16) indicates, we can then complete the analysis by performing a two-dimensional inverse Fourier transform.

We shall use the stochastic transport theory originally introduced by Barabanenkov et al. ${ }^{2}$ and subsequently extended to the two-frequency, absorptive context in Ref. 3. Starting from the stochastic Helmholtz equation, the theory systematically derives a Dyson equation for the mean field $\tilde{u}$ and a radiative transfer equation for a two-frequency complex-valued incoherent intensity function (from which $\left\langle\delta u\left(\mathbf{r}_{1}, k_{1}\right) \delta u^{*}\left(\mathbf{r}_{2}, k_{2}\right)\right\rangle$ can be easily determined). The theory accounts for deterministic refraction and remains valid under conditions of large-angle scattering and large-scale statistical fluctuations. The principal assumptions made are the following: (i) the ratio of difference to sum wavenumbers is small, i.e., $\left|k_{d} / k_{s}\right|<1$, where $k_{d} \equiv k_{1}-k_{2} \quad$ and $k_{s} \equiv\left(k_{1}+k_{2}\right) / 2$; (ii) loss due to true absorption and scattering is small but not negligible; (iii) expectations of certain field quantities and medium parameters vary slowly as a function of the sum variable $\mathbf{R}$ [cf. Eq. (13)].

The derivation of the relevant equations is given in Ref. 3 . We shall simply present the results and comment upon their physical content. Let 


$$
\begin{aligned}
& B(\mathbf{R}, \mathbf{r}) \equiv\left\langle\delta \epsilon\left(\mathbf{R}+\frac{1}{2} \mathbf{r}, k_{0}\right) \delta \epsilon\left(\mathbf{R}-\frac{1}{2} \mathbf{r}, k_{0}\right)\right\rangle, \\
& G_{0}(\mathbf{R}, \mathbf{r}, k) \equiv-\frac{\exp \left[i k\left(\left\langle\epsilon\left(\mathbf{R}, k_{0}\right)\right\rangle\right)^{1 / 2} r\right]}{4 \pi r}, \quad r \equiv|\mathbf{r}|, \\
& M(\mathbf{R}, \mathbf{r}, k) \equiv k^{4} B(\mathbf{R}, \mathbf{r}) G_{0}(\mathbf{R}, \mathbf{r}, k) \equiv M^{\prime}+i M^{\prime \prime}, \\
& \widetilde{M}(\mathbf{R}, \kappa, k) \equiv \int_{R^{3}} M(\mathbf{R}, \mathbf{r}, k) e^{-i \boldsymbol{k} \cdot \mathbf{r}} d \mathbf{r} \equiv \widetilde{M}^{\prime}+i \widetilde{M}^{\prime \prime} .
\end{aligned}
$$

Then, the Dyson equation for the mean or coherent field is given by

$$
\begin{aligned}
{[\Delta+} & \left.k^{2}\left\langle\epsilon\left(\mathbf{r}, k_{0}\right)\right\rangle\right] \tilde{u}(\mathbf{r}, k) \\
& =\int_{R^{3}} M^{\prime}\left(\frac{\mathbf{r}+\mathbf{r}^{\prime}}{2}, \mathbf{r}-\mathbf{r}^{\prime}, k\right) \tilde{u}\left(\mathbf{r}^{\prime}, k\right) d \mathbf{r}^{\prime} .
\end{aligned}
$$

Basically, refraction and absorption caused by the mean dielectric constant arise from terms on the left side of this equation while the dielectric constant fluctuations give rise to the scattering integral on the right.

Before presenting the radiative transfer equation, we must define several quantities. First, we define the effective Hamiltonian ${ }^{9}$

$$
H^{\prime}\left(\mathbf{R}, \kappa, k_{s}\right) \equiv \frac{1}{2}\left\{\kappa^{2}-k_{s}^{2} \operatorname{Re}\left[\left\langle\epsilon\left(\mathbf{R}, k_{0}\right)\right\rangle\right]+\widetilde{M}^{\prime}\left(\mathbf{R}, \kappa, k_{s}\right)\right\},
$$

where $\kappa \equiv|\kappa|, k_{s}=\left(k_{1}+k_{2}\right) / 2$ is the sum wavenumber, and $\widetilde{M}^{\prime}$ is defined in (18). Let $\kappa \equiv \kappa s$. Next, we define an effective wavenumber $k_{e}\left(\mathbf{R}, \mathbf{s}, k_{s}\right)$ as the value of $\kappa$ for which the Hamiltonian vanishes, i.e., $H^{\prime}\left(\mathbf{R}, k_{e} s, k_{s}\right)=0$. Using this effec- tive wavenumber, we introduce an effective index of refraction by the relationship

$$
\left.n_{e}\left(\mathbf{R}, \mathbf{s}, k_{s}\right) \equiv k_{s}^{-1}\left|\nabla_{\kappa} H^{\prime}\left(\mathbf{R}, \kappa \mathrm{s}, k_{s}\right)\right|\right|_{\kappa=k_{e}} .
$$

Finally, we let $\theta\left(\mathbf{R}, \mathbf{s}, k_{s}\right)$ denote the angle between the directions of the group velocity $\left.\nabla_{\kappa} H^{\prime}\left(\mathbf{R}, \kappa \mathrm{s}, k_{s}\right)\right|_{\kappa=k_{e}}$ and the phase velocity $\mathbf{s}$.

The transport equation describes the evolution of a complex-valued, two-frequency, incoherent intensity $I\left(\mathbf{R}, \mathbf{s}, k_{s}, k_{d}\right)$ which is related to the incoherent field fluctuations; specifically,

$$
\begin{aligned}
\left\langle\delta u\left(\mathbf{R}+\frac{1}{2} \mathbf{r}, k_{s}+\frac{1}{2} k_{d}\right) \delta u^{*}\left(\mathbf{R}-\frac{1}{2} \mathbf{r}, k_{s}-\frac{1}{2} k_{d}\right)\right\rangle \\
=\int_{\Omega} k_{s}^{-2} n_{e}^{-3}\left(\mathbf{R}, \mathbf{s}, k_{s}\right) k_{e}^{2}\left(\mathbf{R}, \mathbf{s}, k_{s}\right)\left|\cos \theta\left(\mathbf{R}, \mathbf{s}, k_{s}\right)\right|^{-1} \\
\quad \times I\left(\mathbf{R}, \mathbf{s}, k_{s}, k_{d}\right) \exp \left[i k_{e}\left(\mathbf{R}, \mathbf{s}, k_{s}\right) \mathbf{s} \cdot \mathbf{r}\right] d \mathbf{s},
\end{aligned}
$$

where the integration is taken over the surface of the unit sphere. Let

$$
\begin{aligned}
& \Phi(\mathbf{R}, \mathbf{\kappa}) \equiv \int_{R^{3}} B(\mathbf{R}, \mathbf{r}) e^{-i \kappa \cdot r} d \mathbf{r}, \\
& f_{0}\left(\mathbf{R}, \mathbf{\kappa}, k_{s}, k_{d}\right) \equiv(2 \pi)^{-3} \int_{R^{3}} \tilde{u}\left(\mathbf{R}+\frac{1}{2} \mathbf{r}, k_{s}+\frac{1}{2} k_{d}\right) \tilde{u}^{*} \\
& \times\left(\mathbf{R}-\frac{1}{2} \mathbf{r}, k_{s}-\frac{1}{2} k_{d}\right) e^{-i \boldsymbol{k} \cdot \mathbf{r}} d \mathbf{r} .
\end{aligned}
$$

Then, in terms of the quantities defined in Eqs. (18)-(23), the radiative transfer equation for the incoherent intensity assumes the form

$$
\begin{aligned}
n_{e}^{2} \frac{d}{d l}\left(n_{e}^{-2}|\cos \theta|^{-1} I\right)= & n_{e}^{-1}\left[k_{s}^{-1} \widetilde{M}^{\prime \prime}\left(\mathbf{R}, k_{e} \mathbf{s}, k_{s}\right)-k_{s} \operatorname{Im}\left\{\left\langle\epsilon\left(\mathbf{R}, k_{0}\right)\right\rangle\right\}\right] I+i k_{d} n_{e}^{-1}\left[\operatorname{Re}\left\{\left\langle\epsilon\left(\mathbf{R}, k_{0}\right)\right\rangle\right]\right. \\
& \left.-2 k_{s}^{-2} \widetilde{M}^{\prime}\left(\mathbf{R}, k_{e} \mathbf{s}, k_{s}\right)\right] I+\frac{k_{s}^{2}}{16 \pi^{2}} n_{e}|\cos \theta| \int_{\Omega} k_{e}^{2}\left(\mathbf{R}, \mathbf{s}^{\prime}, k_{s}\right) n_{e}^{-3}\left(\mathbf{R}, \mathbf{s}^{\prime}, k_{s}\right) \\
& \times\left|\cos \theta\left(\mathbf{R}, \mathbf{s}^{\prime}, k_{s}\right)\right|^{-1} \Phi\left[\mathbf{R}, k_{e}\left(\mathbf{R}, \mathbf{s}, k_{s}\right) \mathbf{s}-k_{e}\left(\mathbf{R}, \mathbf{s}^{\prime}, k_{s}\right) \mathbf{s}^{\prime}\right] I\left(\mathbf{R}, \mathbf{s}^{\prime}, k_{s}, k_{d}\right) d \mathbf{s}^{\prime} \\
& +\frac{k_{s}^{4}}{16 \pi^{2}} n_{e}|\cos \theta| \int_{\mathbf{R}^{3}} \Phi\left(\mathbf{R}, k_{e} \mathbf{s}-\boldsymbol{\kappa}^{\prime}\right) f_{0}\left(\mathbf{R}, \boldsymbol{\kappa}^{\prime}, k_{s}, k_{d}\right) d \mathbf{k}^{\prime},
\end{aligned}
$$

where $d l$ denotes a differential at point $\mathbf{R}$ in the direction of the group velocity $\left.\nabla_{\kappa} H^{\prime}\left(R, \kappa \mathrm{s}, k_{s}\right)\right|_{\kappa=k_{e}}$. Unless otherwise noted, arguments in (24) are those appearing in (18)-(23).

The physical interpretation of (24) is straightforward. The left side is a convective term; the ray paths correspond to an effective medium determined both by the deterministic profile and also by the random fluctuations. The first term on the right is a loss term, due both to scattering and to true absorption. The second term is a phase-modifying term that vanishes in the absence of frequency offsets (i.e., in the $\mathrm{cw}$ case). The third term is a scattering integral; the random fluctuations scatter the incoherent intensity in different directions. The last term is a source term, representing the "feeding" of the incoherent intensity by the coherent field. As the wave passes through the random medium, the random fluctuations gradually convert the energy from a coherent to an incoherent state.

\section{SIMPLIFICATIONS DUE TO ANISOTROPY}

The adaptation of this stochastic transport theory to the particular problem being considered will lead to consider- able simplification of the Dyson and transport equations [(19) and (24)]. Noting (15), let

$$
\begin{aligned}
\left\langle\epsilon\left(\mathbf{R}, k_{0}\right)\right\rangle & =\epsilon_{01}\left(k_{0}\right)(1+\eta Z)^{-2}+i \epsilon_{02}\left(\eta Z, k_{0}\right) \\
& \equiv \tilde{\epsilon}\left(\eta Z, k_{0}\right), \quad Z \geqslant 0 .
\end{aligned}
$$

This notation emphasizes the depth dependence and is more concise. One advantage of the dielectric model adopted, i.e., (15), is the ability to explicitly evaluate several integrals of interest. In particular [cf. Eqs. (18), (23)],

$$
\begin{aligned}
& \widetilde{M}\left(\mathbf{R}, \kappa, k_{s}\right)=-\left(k_{s}^{4} / 2\right) B_{0}(\eta Z)\left(\left\{\beta-i k_{s}\left[\epsilon\left(\eta Z, k_{0}\right)\right]^{1 / 2}\right\}^{2}\right. \\
&\left.+\kappa_{T}^{2}\right)^{-1 / 2}, \\
& \Phi(\mathbf{R}, \kappa)=2 \pi \beta B_{0}(\eta Z)\left(\beta^{2}+\kappa_{T}^{2}\right)^{-3 / 2}, \quad \kappa_{T}^{2} \equiv \kappa_{x}^{2}+\kappa_{y}^{2} .
\end{aligned}
$$

Recall that our assumption of severe anisotropy (i.e., large transverse correlation lengths) will permit us to treat $\beta=l_{T}^{-1}$ as a small parameter.

Consider first the Dyson equation for the coherent field [Eq. (19)]. For the pulsed plane-wave excitation being assumed, the field incident upon the water-sediment interface has the form

$$
u_{i}(\mathbf{r}, k)=F(k) \exp \left[i k\left(x \sin \theta_{0}+z \cos \theta_{0}\right)\right],
$$


where $F(k)$ is basically the Fourier transform of the transmitted temporal pulse. For the sediment model being considered, one can then show that the mean or coherent field has the form

$\tilde{u}(\mathbf{r}, k)= \begin{cases}F(k) e^{i k x \sin \theta_{0}}\left[e^{i k z \cos \theta_{0}}+\tilde{r}(k) e^{-i k z \cos \theta_{0}}\right], & z<0, \\ F(k) e^{i k x \sin \theta_{0}} \tilde{v}(z, k), & z \geqslant 0,\end{cases}$

where $\tilde{r}(k)$ is the mean reflection coefficient and $\tilde{v}$ satisfies the following ordinary differential equation:

$$
\begin{aligned}
\frac{d^{2}}{d z^{2}} \tilde{v}(z, k)+ & k^{2} \Omega^{2}(\eta z, k) \tilde{v}(z, k)=0, \quad z \geqslant 0, \\
\Omega^{2}(\eta z, k) \equiv & \tilde{\epsilon}\left(\eta z, k_{0}\right)-\sin ^{2} \theta_{0}+\left(k^{2} / 2\right) B_{0}(\eta z) \\
& \times\left(\left(\beta-i k\left[\tilde{\epsilon}\left(\eta z, k_{0}\right)\right]^{1 / 2}\right\}^{2}\right. \\
& \left.+k^{2} \sin ^{2} \theta_{0}\right)^{-1 / 2} .
\end{aligned}
$$

Therefore, for our problem, the Dyson equation reduces to an ordinary differential equation which in the absence of a refracting profile (i.e., $\eta=0$ ) is a constant coefficient equation. In general, since $\eta$ is a small parameter, (29) is amenable to WKB analysis.

Remark: Equation (29) embodies some very interesting interplay between the refractive and the random scattering mechanisms. Neglect absorption for the present discussion, i.e., assume that $\tilde{\epsilon}$ is real. Then, in the absence of random effects, the turning point occurs at the depth where $\tilde{\epsilon}=\sin ^{2} \theta_{0}$. However, the portion of $\Omega^{2}$ arising from the random fluctuations contains the factor $\left[\beta^{2}-k^{2}\left(\tilde{\epsilon}-\sin ^{2} \theta_{0}\right)-i 2 k \beta \sqrt{\tilde{\epsilon}}\right]^{-1 / 2}$ which peaks sharply at a depth that is slightly less than the nonrandom turning point $\left(\beta^{2}<1\right)$; moreover, the peak magnitude of this factor is $O\left(\beta^{-1 / 2}\right)$. Thus the highly laminated random fluctuations seem to have their greatest local impact upon the mean field in the region near the turning points (where the rays are nearly grazing to the laminates).

The radiative transfer equation (24) also simplifies considerably once the highly anisotropic character of the medium is introduced. Details are given in Ref. 3; we shall only make some comments about the simplification process and then state the result for the special case to be studied in Sec. IV. The following points are worth noting: (i) The structure of (24) is simplified by introducing the new dependent variable

$$
\begin{aligned}
\bar{I}\left(\mathbf{R}, \mathbf{s}, k_{s}, k_{d}\right) \equiv & n_{e}^{-2}\left(\mathbf{R}, \mathbf{s}, k_{s}\right)\left|\cos \theta\left(\mathbf{R}, \mathbf{s}, k_{s}\right)\right|^{-1} \\
& \times I\left(\mathbf{R}, \mathbf{s}, k_{s}, k_{d}\right) ;
\end{aligned}
$$

(ii) When the explicit form for $\Phi$ given by (26) is introduced into the scattering integral in (24) and the smallness of $\beta$ is exploited, the kernel is found to peak sharply at two angles on the unit sphere. These angles correspond to the forwardscattered and specularly reflected directions for the incident ray; (iii) The structure of the mean field $\tilde{u}$ [cf. (28)] and the sharply peaked nature of $\Phi$ lead to considerable simplification of the source integral in (24).

In Sec. IV we shall solve the problem for the special case of no refraction $(\eta=0)$ and normal incidence $\left(\theta_{0}=0\right)$. These simplifications reduce (24) to a linear, constant coefficient, nonhomogeneous, two-dimensional system of ordinary differential equations. The two unknown dependent variables are basically the incoherent energy flowing in the forward and backward depth directions (i.e., into and out of the sediment). More specifically, let

$$
\bar{I}\left(R, \pm \mathrm{z}_{0}, k_{s}, k_{d}\right) \equiv \underset{2}{\tilde{I}_{1}}\left(Z, k_{s}, k_{d}\right) \delta\left(\kappa_{T}\right)
$$

where our notation reflects the fact that in this special case the sum variable dependence of $I$ (and $\bar{I}$ ) involves only the depth variable $Z$. Then, radiative transfer Eq. (24) reduces to

$$
\begin{aligned}
& \frac{d}{d Z} \tilde{I}_{1}=-v \tilde{I}_{1}+\frac{\sigma}{2}\left(\tilde{I}_{1}+\tilde{I}_{2}\right)+\frac{\tilde{s}}{2}, \\
& -\frac{d}{d Z} \tilde{I}_{2}=-v \tilde{I}_{2}+\frac{\sigma}{2}\left(\tilde{I}_{1}+\tilde{I}_{2}\right)+\frac{\tilde{s}}{2}, \quad Z>0,
\end{aligned}
$$

where

$$
\begin{aligned}
& v \equiv-k_{s} k_{e}^{-1}\left[k_{s}^{-1} \widetilde{M}^{\prime \prime}+i k_{d} \epsilon_{01}\left(k_{0}\right)-k_{s} \epsilon_{02}\left(k_{0}\right)\right. \\
& \left.-i 2 k_{d} k_{s}^{-2} \widetilde{M}^{\prime}\right] \text {, } \\
& \sigma \equiv \frac{1}{2} k_{s}^{4} B_{0}\left(\beta^{2}+k_{s}^{2} n_{e}^{2}\right)^{-1} \text {, } \\
& \tilde{s}\left(Z, k_{s}, k_{d}\right) \equiv \frac{1}{2} k_{s}^{5} k_{e}^{-1} B_{0} \tilde{u}\left(Z, k_{s}+\frac{1}{2} k_{d}\right) \tilde{u}^{*}\left(Z, k_{s}-\frac{1}{2} k_{d}\right) \text {. }
\end{aligned}
$$

In the special case being considered, $v$ and $\sigma$ are constants; in the absence of frequency offsets $\left(k_{d}=0\right), v$ is real non-negative. Equations (32) are basically Schuster equations ${ }^{4}$; empirical derivations of one-dimensional transport theory typically lead to equations having this structure. In the absence of frequency offsets, the terms in (32) have the following physical interpretations:

(i) $-v \tilde{I}_{1}$ and $v \tilde{I}_{2}$ represent extinction, i.e., actual dissipation as well as apparent absorption since the random fluctuations "remove" and rescatter the incoherent energy; term;

(ii) $(\sigma / 2)\left(\tilde{I}_{1}+\tilde{I}_{2}\right)$ represents the scattering or reradiation

(iii) $\tilde{s} / 2 \sim B_{0}|\tilde{u}|^{2}$ represents the source term, the mechanism by which coherent energy is converted into incoherent energy.

\section{SOLUTION IN THE SPECIAL CASE OF NO REFRACTION AND NORMAL INCIDENCE}

Assume that a transmitter-receiver at depth $z_{t}$ in the water $\left(. z_{t}<0\right)$ transmits a Gaussian pulsed plane wave. Let $f(t)$ and $\hat{f}(k)$ denote the transmitted temporal pulse and its Fourier transform, respectively. Then, in the water $(z<0)$ the random field of interest is given by

$$
u(z, k)=F(k)\left[e^{i k z}+r(k) e^{-i k z}\right], \quad z<0,
$$

where

$$
\begin{aligned}
& F(k) \equiv \rho_{0}^{-1 / 2} \hat{f}(k) e^{-i k z_{t}}, \\
& r(k)=\langle r(k)\rangle+\delta r(k) \equiv \tilde{r}(k)+\delta r(k) .
\end{aligned}
$$

In the first of Eqs. (35), the factor $\rho_{0}^{-1 / 2}$ arises because $u=\rho^{-1 / 2} \hat{p}$. Using the second of Eqs. (35) we obtain [cf. Eq. (28)]

$$
\begin{aligned}
& \tilde{u}(z, k)=F(k)\left[e^{i k z}+\tilde{r}(k) e^{-i k z}\right], \\
& \delta u(z, k)=F(k) \delta r(k) e^{-i k z}, \quad z<0 .
\end{aligned}
$$

For the special case being considered, we shall be interested in the temporal coherence function of the complex pressure, i.e., $\left\langle p\left(z_{t}, t_{1}\right) p^{*}\left(z_{t}, t_{2}\right)\right\rangle$, where both spatial dependences are fixed at the transceiver depth. We shall introduce sum and difference times as 


$$
t_{s} \equiv\left(t_{1}+t_{2}\right) / 2, t_{d} \equiv t_{1}-t_{2},
$$

and further represent the sum time as

$$
t_{s}=-2 c_{0}^{-1} z_{t}+\tau=2 c_{0}^{-1}\left|z_{t}\right|+\tau
$$

so that $\tau$ measures deviation from the two-way transit time from source to interface. In terms of these quantities we obtain [cf. Eq. (16)]

$$
\begin{aligned}
\left\langle p\left(z_{t}, t_{s}+\frac{1}{2} t_{d}\right) p^{*}\left(z_{t}, t_{s}-\frac{1}{2} t_{d}\right)\right\rangle= & c_{0}^{2} \int_{-\infty}^{\infty} \int_{-\infty}^{\infty} \hat{f}\left(k_{s}+\frac{1}{2} k_{d}\right) \hat{f}^{*}\left(k_{s}-\frac{1}{2} k_{d}\right)\left\{\exp \left[-i k_{s} c_{0} t_{d}-i k_{d}\left(2\left|z_{t}\right|+c_{0} \tau\right)\right]\right. \\
& +\tilde{r}\left(k_{s}+\frac{1}{2} k_{d}\right) \exp \left[-i k_{s}\left(c_{0} t_{d}-2\left|z_{t}\right|\right)-i k_{d}\left(\left|z_{t}\right|+c_{0} \tau\right)\right]+\tilde{r}^{*}\left(k_{s}-\frac{1}{2} k_{d}\right) \\
& \times \exp \left[-i k_{s}\left(c_{0} t_{d}+2\left|z_{t}\right|\right)-i k_{d}\left(\left|z_{t}\right|+c_{0} \tau\right)\right] \\
& +\left[\tilde { r } \left(k_{s}+\frac{1}{2} k_{d} \mid \tilde{r}^{*}\left(k_{s}-\frac{1}{2} k_{d}\right)+\left\langle\delta r\left(k_{s}+\frac{1}{2} k_{d}\right) \delta r^{*}\left(k_{s}-\frac{1}{2} k_{d}|\rangle\right]\right.\right.\right. \\
& \left.\times \exp \left[-i k_{s} c_{0} t_{d}-i k_{d} c_{0} \tau\right]\right\} d k_{s} d k_{d} .
\end{aligned}
$$

Of the four bracketed terms in (39), only the last has significance with respect to modeling measured data. The first term involves the incident wave while the next two terms involve products of incident and reflected waves. With $\left|z_{t}\right|$ large, these terms are eliminated by time-gating. The quantity of interest is;

$$
\begin{aligned}
I\left(\tau, t_{d}\right) \equiv & c_{0}^{2} \int_{-\infty}^{\infty} \int_{-\infty}^{\infty} \hat{f}^{*}\left(k_{s}+\frac{1}{2} k_{d}\right) \hat{f}\left(k_{s}-\frac{1}{2} k_{d}\right)\left[\tilde{r}\left(k_{s}+\frac{1}{2} k_{d}\right) r\left(k_{s}-\frac{1}{2} k_{d}\right)+\left\langle\delta r\left(k_{s}+\frac{1}{2} k_{d}\right) \delta r^{*}\right.\right. \\
& \left.\left.\times\left(k_{s}-\frac{1}{2} k_{d}\right)\right)\right] \exp \left[-i k_{s} c_{0} t_{d}-i k_{d} c_{0} \tau\right] d k_{s} d k_{d} .
\end{aligned}
$$

In order to determine $I\left(\tau, t_{d}\right)$ we shall first solve for the coherent field $\tilde{u}$ (and therefore determine $\tilde{r}$ ). Once $\tilde{u}$ is known, the source term in (32) is specified. We shall then solve for the incoherent intensity and from this information determine $\left\langle\delta r\left(k_{s}+\frac{1}{2} k_{d}\right) \delta r^{*}\left(k_{s}-\frac{1}{2} k_{d}\right)\right\rangle$. The two-dimensional transform in (40) completes the computation.

Although the equations in this case are very tractable, i.e., constant coefficient ordinary differential equations [(29), (32)], interface boundary conditions must be formulated to complete the problem specifications. Such boundary conditions are not generated as part of the transport theory derivation since this analysis invariably assumes an infinite medium in which mean quantities vary slowly. In our problem, the task is complicated by the fact that (i) the mean density and sound speed are generally discontinuous across the interface, and (ii) in the two-frequency case $\left(k_{d} \neq 0\right)$, the incoherent intensity is complex-valued and cannot be interpreted as an energy. We shall now digress and briefly consider this important topic.

\section{A. Interface boundary conditions}

For each random realization, pressure and normal component of velocity must be continuous across the interface. Recall that $u=\rho^{-1 / 2} \hat{p}$. The task at hand is to circumvent the closure problem and derive appropriate boundary conditions for $\langle u(\mathbf{r}, k)\rangle=\tilde{u}(\mathbf{r}, k)$ and $\left\langle u\left(\mathbf{r}, k_{1}\right) u^{*}\left(\mathbf{r}, k_{2}\right)\right\rangle$.

For the mean field, we first note that for each random realization, $\rho^{1 / 2} u$ and $\rho^{-1}(\partial / \partial z)\left(\rho^{1 / 2} u\right)$ must be continuous across $z=0$. Taking averages and using a perturbation analysis based on the smallness of $\delta \tilde{\rho}$ leads to the boundary conditions:

$$
\begin{aligned}
& \left.\tilde{u}\right|_{z=0^{-}}=\left.\tilde{\rho}_{1}^{1 / 2} \tilde{u}\right|_{z=0^{+}}, \\
& \left.\frac{\partial}{\partial z} \tilde{u}\right|_{z=0^{-}}=\left.\tilde{\rho}_{1}^{-1 / 2} \frac{\partial}{\partial z} \tilde{u}\right|_{z=0^{+}}
\end{aligned}
$$

To obtain boundary conditions for the second moments, we follow the approach of Keller ${ }^{5}$ and define a two-frequency intensity and flux

$$
\begin{aligned}
\mathscr{I}\left(\mathbf{r} ; k_{1}, k_{2}\right) \equiv & u\left(\mathbf{r}, k_{1}\right) u^{*}\left(\mathbf{r}, k_{2}\right), \\
\mathscr{S}\left(\mathbf{r} ; k_{1}, k_{2}\right) \equiv & (1 / 2 i)\left[u^{*}\left(\mathbf{r}, k_{2}\right) \nabla u\left(\mathbf{r}, k_{1}\right)\right. \\
& \left.-u\left(\mathbf{r}, k_{1}\right) \nabla u^{*}\left(\mathbf{r}, k_{2}\right)\right],
\end{aligned}
$$

where $u$ is a solution of the random Helmholtz Eq. (9). The intensity and flux satisfy an equation of continuity; specifically,

$$
\nabla \cdot \mathscr{S}+(1 / 2 i)\left[k_{1}^{2} \epsilon\left(\mathbf{r}, k_{1}\right)-k_{2}^{2} \epsilon^{*}\left(\mathbf{r}, k_{2}\right)\right] \mathscr{I}=0 .
$$

For each realization, the second term in (43) undergoes a jump discontinuity at worst across the interface. Using the Divergence Theorem and the standard "pillbox" argument, we infer that $\mathscr{S} \cdot \mathrm{z}_{0}$ is continuous across $z=0$; hence, $\langle\mathscr{S}\rangle \cdot \mathbf{z}_{\mathbf{0}}$ is continuous across the interface. The boundary condition for $\langle\mathscr{I}\rangle$ can be obtained using the same ideas that led to (41). The resulting pair of boundary conditions is

$$
\begin{aligned}
& \left.\langle\mathscr{S}\rangle\right|_{z=0^{-}}=\left.\tilde{\rho}_{1}\langle\mathscr{S}\rangle\right|_{z=0^{+}}, \\
& \left.\langle\mathscr{S}\rangle \cdot \mathbf{z}_{0}\right|_{z=0^{-}}=\left.\langle\mathscr{S}\rangle \cdot \mathbf{z}_{0}\right|_{z=0^{+}} .
\end{aligned}
$$

Let us define coherent and incoherent average intensities and fluxes as follows:

$$
\begin{gathered}
\tilde{\mathscr{I}}_{c}\left(\mathbf{r} ; k_{1}, k_{2}\right) \equiv \tilde{u}\left(\mathbf{r}, k_{1}\right) \tilde{u}^{*}\left(\mathbf{r}, k_{2}\right), \\
\tilde{\mathscr{I}}_{i} \equiv\langle\mathscr{I}\rangle-\tilde{\mathscr{I}}_{c}, \\
\tilde{\mathscr{S}}_{c}\left(\mathbf{r} ; k_{1}, k_{2}\right) \equiv(1 / 2 i)\left[\tilde{u}^{*}\left(\mathbf{r}, k_{2}\right) \nabla \tilde{u}\left(\mathbf{r}, k_{1}\right)\right. \\
\left.\quad-\tilde{u}\left(\mathbf{r}, k_{1}\right) \nabla \tilde{u}^{*}\left(\mathbf{r}, k_{2}\right)\right], \\
\tilde{\mathscr{S}}_{i} \equiv\langle\mathscr{S}\rangle-\tilde{\mathscr{S}}_{c} .
\end{gathered}
$$

Using (41), one can show that $\tilde{\mathscr{I}}_{c}$ and $\tilde{\mathscr{S}}_{c}$ satisfy boundary conditions (44). Therefore the coherent and incoherent average intensities and fluxes individually satisfy boundary conditions (44).

The problem for the mean or coherent field is now de- 
fined by Eqs. (28), (29) (with $\theta_{0}=0, \eta=0$ ) and boundary conditions $(41)$. Note that $\Omega^{2}$ is now constant. One readily finds that

$$
\begin{aligned}
& \tilde{u}(z, k)=F(k)\left[2 \tilde{\rho}_{1}^{1 / 2} /\left(\tilde{\rho}_{1}+\Omega\right)\right] e^{i k \Omega z}, \quad z>0, \\
& \tilde{r}(k)=\left(\tilde{\rho}_{1}-\Omega\right) /\left(\tilde{\rho}_{1}+\Omega\right),
\end{aligned}
$$

with $\operatorname{Im}\{\Omega\}>0$.

The problem for the incoherent fluctuations [or equivalently $\left.\left\langle\delta r\left(k_{1}\right) \delta r^{*}\left(k_{2}\right)\right\rangle\right]$ is defined by (32) and boundary conditions (44). Note that the incoherent intensity and flux can be expressed as

$$
\begin{aligned}
\tilde{\mathscr{I}}_{i}\left(\mathbf{r}, k_{1}, k_{2}\right)= & \left\langle\delta u\left(\mathbf{r}, k_{1}\right) \delta u^{*}\left(\mathbf{r}, k_{2}\right)\right\rangle, \\
\tilde{\mathscr{S}}_{i}\left(\mathbf{r}, k_{1}, k_{2}\right)= & \frac{1}{2 i}\left[\nabla_{\mathbf{r}_{1}}-\nabla_{\mathbf{r}_{2}}\right]\left\langle\delta u\left(\mathbf{r}_{1}, k_{1}\right) \delta u^{*}\right. \\
& \left.\times\left(\mathbf{r}_{2}, k_{2}\right)\right\rangle\left.\right|_{\mathbf{r}_{1}=\mathbf{r}_{2}=\mathbf{r}} .
\end{aligned}
$$

Boundary conditions (44) hold separately for these incoherent quantities. In terms of the sum and difference variables, the use of (36) in (47) leads to

$$
\begin{aligned}
\tilde{\mathscr{I}}_{i}\left(Z, k_{s}+\frac{1}{2} k_{d}, k_{s}-\frac{1}{2} k_{d}\right)= & F\left(k_{s}+\frac{1}{2} k_{d}\right) F^{*}\left(k_{s}-\frac{1}{2} k_{d}\right) \\
& \times\left\langle\delta r ( k _ { s } + \frac { 1 } { 2 } k _ { d } ) \delta r ^ { * } \left( k_{s}\right.\right. \\
& \left.\left.-\frac{1}{2} k_{d}\right)\right\rangle e^{-i k_{d} Z}, \\
\tilde{\mathscr{S}}_{i}\left(Z, k_{s}+\frac{1}{2} k_{d}, k_{s}-\frac{1}{2} k_{d}\right)= & -k_{s} F\left(k_{s}+\frac{1}{2} k_{d}\right) F^{*} \\
& \times\left(k_{s}-\frac{1}{2} k_{d}\right)\left\langle\delta r\left(k_{s}+\frac{1}{2} k_{d}\right),\right. \\
& \times \delta r^{*}\left(k_{s}-\frac{1}{2} k_{d}\right\rangle e^{-i k_{d} Z} \mathbf{z}_{0},
\end{aligned}
$$

in $Z<0$ (i.e., the water region). The notational changes in (48) emphasize the sum depth dependence. Note that the incoherent flux is directed in the $-z_{0}$ direction (i.e., out of the sediment and into the water.) In the sediment region, (22), (30), (31), and (32) can be used to express the incoherent intensity and flux as

$$
\begin{aligned}
\tilde{\mathscr{I}}_{i}\left(Z, k_{s}+\frac{1}{2} k_{d}, k_{s}-\frac{1}{2} k_{d}\right)= & k_{s}^{-2} n_{e}^{-1}\left[\tilde{I}_{1}\left(Z, k_{s}, k_{d}\right)\right. \\
& \left.+\tilde{I}_{2}\left(Z, k_{s}, k_{d}\right)\right], \\
\widetilde{\mathscr{S}}_{i}\left(Z, k_{s}+\frac{1}{2} k_{d}, k_{s}-\frac{1}{2} k_{d}\right)= & k_{s}^{-2} n_{e}^{-1} k_{e}\left[\tilde{I}_{1}\left(Z, k_{s}, k_{d}\right)\right. \\
& \left.-\tilde{I}_{2}\left(Z, k_{s}, k_{d}\right)\right] \mathrm{z}_{0},
\end{aligned}
$$

in $Z>0$. [As part of the derivation of (49), differential equation (32) was used to related $/ d Z\left(\tilde{I}_{1}+\tilde{I}_{2}\right)$ to $\left.\left(\tilde{I}_{1}-\tilde{I}_{2}\right)\right]$. Applying boundary conditions (44) to (48), (49) leads to the representation:

$$
\begin{gathered}
F\left(k_{s}+\frac{1}{2} k_{d}\right) F^{*}\left(k_{s}-\frac{1}{2} k_{d}\right)\left\langle\delta r\left(k_{s}+\frac{1}{2} k_{d}\right) \delta r^{*}\left(k_{s}-\frac{1}{2} k_{d}\right)\right\rangle \\
=-k_{s}^{-3} n_{e}^{-1} k_{e}\left[\Gamma\left(k_{s}, k_{d}\right)-1\right] \tilde{I}_{2}\left(0^{+}, k_{s}, k_{d}\right), \\
\Gamma\left(k_{s}, k_{d}\right) \equiv \tilde{I}_{1}\left(0^{+}, k_{s}, k_{d}\right) / \tilde{I}_{2}\left(0^{+}, k_{s}, k_{d}\right) .
\end{gathered}
$$

To determine $\tilde{I}_{2}\left(0^{+}, k_{s}, k_{d}\right)$, we solve differential equation (32) in $Z>0$, imposing the proper boundary condition as $Z \rightarrow \infty$. This boundary condition was conveniently ascertained by introducing a rock basement, solving the problem for this configuration and subsequently letting the sediment-basement interface recede to infinity. The result is

$$
\tilde{I}_{2}\left(0^{+}, k_{s}, k_{d}\right)=\frac{\left\{v-[v(v-\sigma)]^{1 / 2}\right\} \int_{0}^{\infty} \tilde{s}(\xi) \exp \left(-[v(v-\sigma)]^{1 / 2} \xi\right) d \xi}{\sigma[1-\Gamma]+2 \Gamma[v(v-\sigma)]^{1 / 2}},
$$

where $v, \sigma, \tilde{s}$ are defined in (33) and (46).

Using (51) in (50) enables us to specify the reflection coefficient fluctuations $\left\langle\delta r\left(k_{s}+\frac{1}{2} k_{d}\right) \delta r^{*}\left(k_{s}-\frac{1}{2} k_{d}\right)\right\rangle$. Combining this result with (46), the quantity of interest $I\left(\tau, t_{d}\right)$ [cf. Eq. (40)] can be expressed as

$$
\begin{aligned}
I\left(\tau, t_{d}\right)= & c_{0}^{2} \int_{-\infty}^{\infty} \int_{-\infty}^{\infty} \hat{f}\left(k_{s}+\frac{1}{2} k_{d}\right) \hat{f}^{*}\left(k_{s}-\frac{1}{2} k_{d}\right) \exp \left(-i k_{s} c_{0} t_{d}-i k_{d} c_{0} \tau\right)\left[\left(\frac{1-\tilde{\rho}_{1}^{-1} \Omega\left(k_{s}+\frac{1}{2} k_{d}\right)}{1+\tilde{\rho}_{1}^{-1} \Omega\left(k_{s}+\frac{1}{2} k_{d}\right)}\right)\right. \\
& \times\left(\frac{1-\tilde{\rho}_{1}^{-1} \Omega^{*}\left(k_{s}-\frac{1}{2} k_{d}\right)}{1+\tilde{\rho}_{1}^{-1} \Omega^{*}\left(k_{s}-\frac{1}{2} k_{d}\right)}\right)+2 k_{s}^{2} n_{e}^{-2} B_{0}\left\{v-[v(v-\sigma)]^{1 / 2}\right\}\left\{\sigma \tilde{\rho}_{1} n_{e}^{-1}+\left(1-\tilde{\rho}_{1} n_{e}^{-1}\right)[v(v-\sigma)]^{1 / 2}\right\}^{-1} \\
& \times\left\{1+\tilde{\rho}_{1}^{-1} \Omega\left(k_{s}+\frac{1}{2} k_{d}\right)\right\}^{-1}\left\{1+\tilde{\rho}_{1}^{-1} \Omega^{*}\left(k_{s}-\frac{1}{2} k_{d}\right)\right\}^{-1}\left\{[v(v-\sigma)]^{1 / 2}-i\left(k_{s}+\frac{1}{2} k_{d}\right) \Omega\left(k_{s}+\frac{1}{2} k_{d}\right)\right. \\
& \left.\left.+i\left(k_{s}-\frac{1}{2} k_{d}\right) \Omega *\left(k_{s}-\frac{1}{2} k_{d}\right)\right\}^{-1}\right] d k_{s} d k_{d} .
\end{aligned}
$$

\section{NUMERICAL EVALUATION AND QUALITATIVE COMPARISON WITH DATA}

Equation (52) was evaluated numerically; where possible, parameter values were chosen to correspond to data taken at the Hatteras abyssal plain. ${ }^{10}$ The following values were adopted:

Density in water: $\rho_{0}=1.05 \mathrm{~g} / \mathrm{cm}^{3}$.

Sound speed in water: $c_{0}=1550 \mathrm{~m} / \mathrm{s}$.

Mean density in sediment: $\rho_{1}=1.62 \mathrm{~g} / \mathrm{cm}^{3}$.

Mean sound speed in sediment: $c_{1}=1582 \mathrm{~m} / \mathrm{s}$.

Normalized density fluctuations [cf. (12)]: $\sigma_{\rho \rho}$ $\cong 0.0185$.

Normalized sound-speed fluctuations: $\sigma_{c c}$ $\cong 0.0025$.
Sediment correlation lengths: $l_{T}=\beta^{-1}=10 \mathrm{~m}$ (a guess), $l_{z} \cong 0.2 \mathrm{~m}$.

Sediment absorption: $\epsilon_{02} \cong 0.001$.

Transmitted signal: $f(t)=(1 / \sqrt{2 \pi} T) \exp \left[-t^{2} /\left(2 T^{2}\right)\right.$

$+i \omega_{0} t$, with $T=10 \mathrm{~ms}$ and $1 \mathrm{kHz} \leqslant f_{0} \leqslant 4 \mathrm{kHz}$.

Here, approximate equality denotes a nominal value that was varied parametrically.

$I(\tau, 0)\left[i . e .,(52)\right.$ with $t_{d}=0$ ] was studied since this quantity was also measured experimentally. However, since the measurements were made at a grazing angle of $45^{\circ}$ with Gaussian beams, comparison of theory and experiment is qualitative at best.

The numerical study of $I(\tau, 0)$ led to the following observations ${ }^{11}$ : (a) Decreasing the size of the sediment density and 

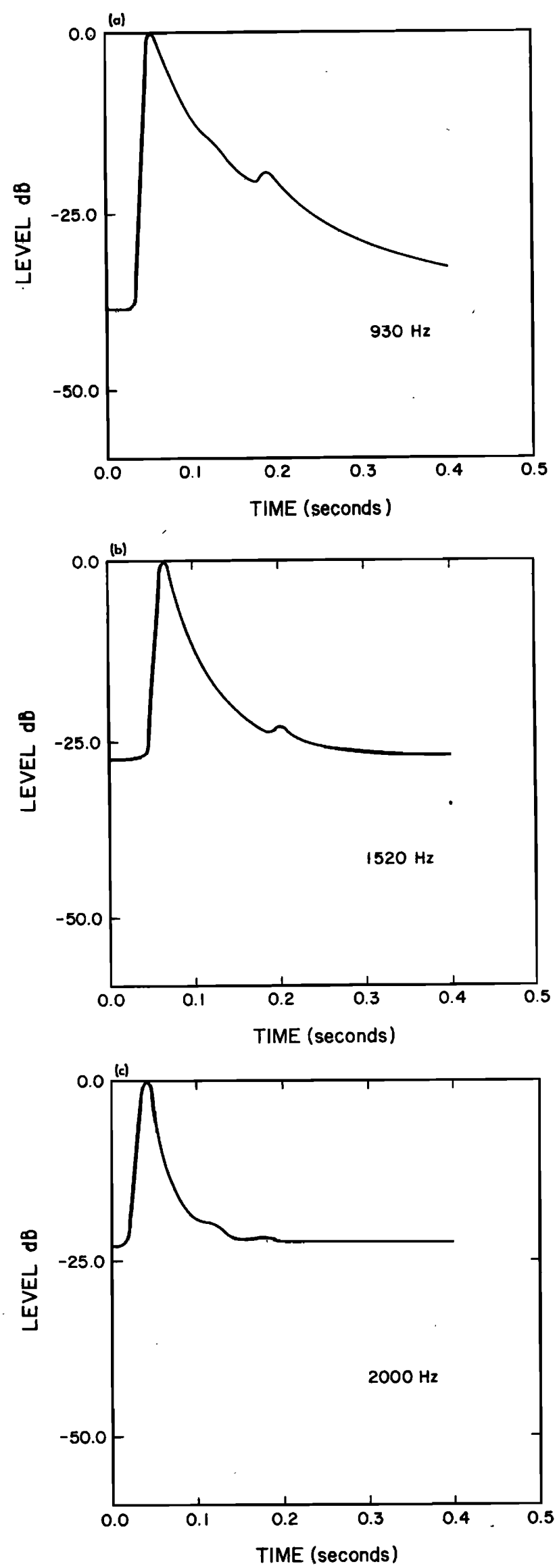

FIG. 2. Experimental measurements of $I(\tau, 0)\left(45^{\circ}\right.$ grazing angle, Gaussian beam antenna) obtained from averages of several thousand pulses along a 12-mile track at three center frequencies: (a) $930 \mathrm{~Hz}$; (b) $1520 \mathrm{~Hz}$; (c) 2000 Hz. The second small peaks correspond to bottom-surface paths.
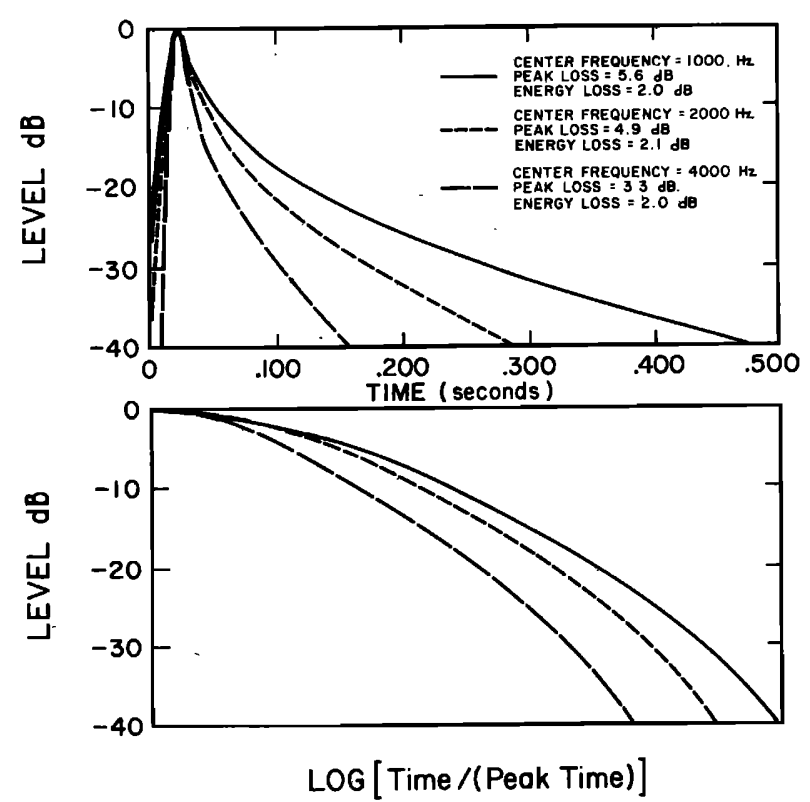

FIG. 3. Theoretical evaluation of $I(\tau, 0)\left(90^{\circ}\right.$ grazing angle, no profile, planewave incidence). Pulse length $=0.005 \mathrm{~s}(\mathrm{rms})$, sound speed in water $=1550$ $\mathrm{m} / \mathrm{s}$, density in water $=1.05$. Sound speed in sediment $=1582 \mathrm{~m} / \mathrm{s}$, density in sediment $=1.62$, dissipation constant in sediment $=0.0010$. Density variance $=0.01850$, sound speed variance $=0.00250$, density $/$ sound speed covariance $=0.00680$, transverse scale size $=10.000$, vertical scale size $=0.200$. Analysis bandwidth $=400 \mathrm{~Hz}$, grazing angle $=90^{\circ}$.

sound-speed fluctuations results in slower pulse decay rates and increasing loss. This appears due to the ability of the pulse to penetrate more deeply into the sediment; (b) decreasing the vertical scale size of the sediment density and soundspeed fluctuations results in a faster pulse decay rate. The effect on pulse energy, however, does not show a consistent pattern; (c) the decay rates in all cases are slower than exponential (but faster than the experimentally observed algebraic rates); (d) decreasing background dissipation increases pulse duration and decreases loss; (e) behavior as a function of frequency is more complicated. For certain parameter values (including vertical scale sizes on the order of $0.2 \mathrm{~m}$ ) the pulse decay rates increase with increasing frequency in the manner observed experimentally. Figures 2 and 3 illustrate this behavior. On the other hand, for vertical scale sizes on the order of $0.05 \mathrm{~m}$ the behavior is exactly the opposite; the decay rates decrease with increasing frequency. However, as the fluctuation amplitudes decrease at constant vertical scale size, the decay rate behavior goes from the latter to former -type of behavior, suggesting that the changeover region is a function of the ratio of fluctuation amplitude to vertical scale size.

\section{CONCLUSIONS}

Some new but still preliminary inroads into the problem of pulse propagation through sediments have been made. Virtually every aspect of our discussion requires deeper understanding and generalization. Of particular importance is the need to properly account for refraction. More realistic modeling of the refractive index and incident radiation also seem to be important needs. Once these major points have been resolved, we believe that it will be possible to incorpo- 
rate other realistic features (e.g., surface roughness and large-scale laminate undulations) into the model without inordinate difficulty.

\section{ACKNOWLEDGMENTS}

The work was performed under U. S. Office of Naval Research Contrct No. N00014-76-C-0056P00004 and with funding from NAUSEA Code 63D. Work on this problem was motivated by a lecture given by $\mathrm{A}$. Ellinthorpe. ${ }^{12}$

\section{APPENDIX A: OBLIQUE INCIDENCE; NO REFRACTION}

In the absence of refraction (i.e., $\eta=0$ ), extension of the results to oblique incidence angles $\left(\theta_{0} \neq 0\right)$ is straightforward. Equation (40) remains valid if we correctly redefine $\tilde{r}(k)$ and $\left\langle\delta r\left(k_{1}\right) \delta r^{*}\left(k_{2}\right)\right\rangle$. For oblique incidence,

$$
r(k)=\left[\cos \theta_{0}-\tilde{\rho}_{1}^{-1} \Omega(k)\right] /\left[\cos \theta_{0}+\tilde{\rho}_{1}^{-1} \Omega(k)\right],
$$

where, as before, $\Omega(k)$ denotes theappropriate root of $\Omega^{2}(0, k)$ [cf. Eq. (29)]. The reflection factor fluctuations are given by

$$
\begin{aligned}
\left\langle\delta r \left( k_{s}\right.\right. & \left.\left.+\frac{1}{2} k_{d}\right) \delta r^{*}\left(k_{s}-\frac{1}{2} k_{d}\right)\right) \\
= & 2 k_{s}^{3} k_{e}^{-1} n_{e}^{-1} B_{0}\left\{v-[v(v-\sigma)]^{1 / 2}\right\} \cos ^{2} \theta_{0} \cos ^{2} \theta \sec \psi\left(\frac{\beta+k_{e} \sin \theta_{0}+(2 / \pi) \tan ^{-1}\left(k_{e} \beta^{-1} \sin \theta_{0}\right)}{\left(\beta^{2}+k_{e}^{2} \sin ^{2} \theta_{0}\right)^{1 / 2}}\right) \\
& \times\left\{\sigma \tilde{\rho}_{1} k_{s} k_{e}^{-1} \cos \theta_{0} \sec \psi+\left(1-\tilde{\rho}_{1} k_{s} k_{e}^{-1} \cos \theta_{0} \sec \psi\right)[v(v-\sigma)]^{1 / 2}\right\}^{-1}\left\{\cos \theta_{0}+\tilde{\rho}_{1}^{-1} \Omega\left(k_{s}+\frac{1}{2} k_{d}\right)\right\}^{-1} \\
& \times\left\{\cos \theta_{0}+\tilde{\rho}_{1}^{-1} \Omega^{*}\left(k_{s}-\frac{1}{2} k_{d}\right)\right\}^{-1}\left\{[v(v-\sigma)]^{1 / 2}-i\left(k_{s}+\frac{1}{2} k_{d}\right) \Omega\left(k_{s}+\frac{1}{2} k_{d}\right)+i\left(k_{s}-\frac{1}{2} k_{d}\right) \Omega *\left(k_{s}-\frac{1}{2} k_{d}\right)\right\}^{-1},
\end{aligned}
$$

where the terms in (A2) are defined as follows:

$$
\begin{aligned}
& k_{e}=k_{s}\left\{\operatorname{Re}\left[\Omega\left(k_{s}\right)\right]\right\}^{1 / 2}, \\
& \sin \psi=k_{s} k_{e}^{-1} \sin \theta_{0} \text {, } \\
& \Lambda \equiv 1+\frac{1}{4} B_{0} \operatorname{Re}\left\{\left[\left(\beta-i k_{s} \sqrt{\tilde{\epsilon}}\right)^{2}+k_{s}^{2} \sin ^{2} \theta_{0}\right]^{-3 / 2}\right\} \text {, } \\
& \cos \theta=\left[\cos ^{2} \psi+\Lambda \sin ^{2} \psi\right] /\left[\cos ^{2} \psi+\Lambda^{2} \sin ^{2} \psi\right]^{1 / 2} \geqslant 0, \\
& n_{e}=k_{s}{ }^{-1} k_{e}\left(\cos ^{2} \psi+\Lambda^{2} \sin ^{2} \psi\right)^{1 / 2} \text {, } \\
& \widetilde{\boldsymbol{M}}=\widetilde{\boldsymbol{M}}^{\prime}+i \widetilde{\boldsymbol{M}}^{\prime \prime}=-\frac{1}{2} k_{s}^{4} B_{0}\left[\left(\beta-i k_{s} \sqrt{\tilde{\epsilon}}\right)^{2}+k_{s}^{2} \sin ^{2} \theta_{0}\right]^{-1 / 2} \text {, } \\
& v=k_{s} k_{e}^{-1} \sec \psi\left[i k_{d} \sin ^{2} \theta_{0}\left(1+\frac{1}{2} k_{s}^{4} B_{0} \operatorname{Re}\left(\left[\beta-i k_{s} \sqrt{\tilde{\epsilon}}\right)^{2}+k_{s}^{2} \sin ^{2} \theta_{0}\right]^{-3 / 2}\right\}\right) \\
& \left.-\cos \theta\left(k_{s}^{-1} \widetilde{M}^{\prime \prime}+i k_{d} \epsilon_{01}-k_{s} \epsilon_{02}-i 2 k_{d} k_{s}^{-2} \widetilde{M}^{\prime}\right)\right] \text {, } \\
& \sigma=\frac{1}{2} k_{s}^{3} k_{e} n_{e}^{-1} \sec \psi \cos \theta B_{0}\left[\beta^{2}+4 k_{e}^{2} \sin ^{2} \psi\right]^{-1 / 2}\left[2 k_{e}^{-1} \tan \psi+\beta^{2} \cos \psi\left(\beta^{2}+k_{e}^{2} \cos ^{2} \psi\right)^{-1}\right. \\
& \left.\times\left(\frac{\cos \psi}{\left(\beta^{2}+k_{e}^{2} \sin ^{2} \psi \cos ^{2} \psi\right)^{1 / 2}}-\frac{\sin \psi}{\left(\beta^{2}+k_{e}^{2} \cos ^{4} \psi\right)^{1 / 2}}\right)\right] .
\end{aligned}
$$

As $\theta_{0}$ approaches $0, \theta$ and $\psi$ likewise approach 0 ; we recover (52) in this limit.

\section{APPENDIX B: ENERGY CONSERVATION}

Consider Eqs. (40) and (52). A measure of the reflected acoustic energy is given by

$$
Q=\int_{-\infty}^{\infty} I(\tau, 0) d \tau
$$

Using (40) and interchanging orders of integration, we obtain

$$
Q=c_{0} \int_{-\infty}^{\infty}\left|\hat{f}\left(k_{s}\right)\right|^{2}\left[\left|\tilde{r}\left(k_{s}\right)\right|^{2}+\left\langle\left|\delta r\left(k_{s}\right)\right|^{2}\right\rangle\right]
$$

One can verify, using (52), that

$$
\left|\tilde{r}\left(k_{s}\right)\right|^{2}+\left\langle\left|\delta r\left(k_{s}\right)\right|^{2}\right\rangle \leqslant 1,
$$

so that

$$
Q \leqslant \int_{-\infty}^{\infty}|\hat{f}(k)|^{2} d k=\int_{-\infty}^{\infty}|f(t)|^{2} d t
$$

${ }^{1}$ E. L. Hamilton, "Geoacoustic Models of the Sea Floor," in Physics of Sound in Marine Sediments, edited by L. Hampton (Plenum, New York, 1974), pp. 181-221.
${ }^{2}$ Y. N. Barabanenkov, A. G. Vinogradov, Y. A. Kravtsov, and V. I. Tatarskii, "Application of the Theory of Multiple Scattering of Waves to the Derivation of the Radiation Transfer Equation for a Statistically Inhomogeneous Medium," Izv. Vyssh. Uchebn, Zaved., Radiofiz. 15, 1852 (1972). ${ }^{3}$ I. M. Besieris and W. E. Kohler, "Two-Frequency Radiative Transfer Equation for a Statistically Inhomogeneous and Anisotropic Absorptive Medium," Proc. Symp. on Multiple Scattering and Waves in Random Media, edited by P. L. Chow, W. Kohler, and G. Papanicolaou (North-Holland, Amsterdam, 1981).

${ }^{4}$ A. Schuster, "Radiation Through a Foggy Atmosphere," Astrophys. J. 21, 1-22 (1905).

${ }^{5}$ J. B. Keller, "Stochastic Equations and Wave Propagation in Random Media," Proc. Symp. Appl. Math. 16, 145-170 (1964).

${ }^{6}$ L. M. Brekhovskikh, Waves in Layered Media (Academic, New York, 1960).

'S. K. Mitchel and K. C. Focke, "New Measurements of Compressional Wave Attenuation in Deep Ocean Sediments," J. Acoust. Soc. Am. 67, 1582-1589 (1980).

${ }^{8}$ B. E. Tucholke, "Acoustic Environment of the Hatteras and Nares Abyssal Plains, Western North Atlantic Ocean, Determined from Velocities and Physical Properties of Sediment Cores," J. Acoust. Soc. Am. 68, 1376-1390 (1980).

${ }^{9} H^{\prime}\left(\mathbf{R}, \kappa, k_{s}\right)$ in Eq. (20) is the real part of the complete effective Hamiltonian $H\left(\mathbf{R}, \kappa, k_{s}\right)$ associated with the mean field $\tilde{u}$. For $z \geqslant 0$, the latter has the form $H\left(\mathbf{R}, \kappa, k_{s}\right)=H^{\prime}\left(\mathbf{R}, \kappa, k_{s}\right)+i H^{\prime \prime}\left(\mathbf{R}, \kappa, k_{s}\right)=(1 / 2)\left[\kappa^{2}-k_{s}^{2}\left\langle\epsilon\left(\mathbf{R}, k_{0}\right)\right\rangle\right.$ 
$\left.+\widetilde{M}\left(\mathbf{R}, \kappa, k_{s}\right)\right]$, where $\left\langle\epsilon\left(\mathbf{R}, k_{0}\right)\right\rangle=\epsilon_{01}\left(k_{0}\right)(1+\eta Z)^{-2}+i \epsilon_{02}\left(\eta Z, k_{0}\right)[c f$. Eq. (15)] and $\widetilde{M}\left(\mathbf{R}, \kappa, k_{s}\right)=\widetilde{M}^{\prime}\left(\mathbf{R}, \kappa, k_{s}\right)+i \widetilde{M}\left(\mathbf{R}, \kappa, k_{s}\right)$. The Hamiltonian $H\left(R, \kappa, k_{s}\right)$ describes an effective medium whose regular refraction $\operatorname{Re}\left[\left\langle\epsilon\left(\mathbf{R}, k_{0}\right)\right\rangle\right]$ is augmented by $\left(1 / k_{s}{ }^{2}\right) \widetilde{M}^{\prime}\left(\mathbf{R}, \kappa, k_{s}\right)$ and whose regular loss $\left.\operatorname{Im}\left\{\left\langle\epsilon \mathbf{R}, k_{0}\right)\right\rangle\right]$ is supplemented by the scattering loss $\left(1 / k_{s}^{2}\right) \tilde{M} "\left(R, \kappa, k_{s}\right)$. An insistence on using the exact Hamiltonian $H\left(R, \kappa, k_{s}\right)$ instead of $H^{\prime}\left(R, \kappa, k_{s}\right)$ would yield a radiative transport theory based on complex rather than real rays. Although such a procedure is feasible in principle, it will be avoided in the following development by assuming that the regular and scattering loss terms $k_{s}^{2} \operatorname{Im}\left\{\left\langle\epsilon\left(\mathbf{R}, k_{0}\right)\right\rangle\right], \widetilde{M}^{\prime \prime}\left(\mathbf{R}, \kappa, k_{s}\right)$ are small, but not negligible.

${ }^{10} \mathrm{H}$. Freese, "Impulse Response Decay Rates of Single Bottom Bounce Paths," Naval Underwater Systems Center/New London Laboratory Memo. Ser. 83103-68 (17 July 1978).

"H. Freese, "Evaluation of W. Kohler's Radiative Transport Equations for Vertical Incidence Backscatter," Naval Underwater Systems Center/ New London Laboratory Memo. Ser. 13292-11 (5 May 1981).

${ }^{12}$ A. Ellinthorpe, "Some Propagation Statistics Attributable to Bottom Interaction," a lecture presented at a Seminar on Bottom Effects in Underwater Sound Propagation, Miami, FL (April 1979). 